\title{
CME Tropical medicine SAQs (91578): Self-assessment questionnaire
}

\author{
Edited by Cordelia Coltart and Tahseen Chowdhury
}

\section{SAQs and answers are ONLINE for RCP fellows and collegiate members}

The SAQs printed in the CME section can only be answered online to achieve external CPD credits. Any comments should be sent in via email only: clinicalmedicine@rcplondon.ac.uk

\section{Format}

SAQs follow a best of five format in line with the MRCP(UK) Part 1 exam. Candidates are asked to choose the best answer from five possible answers.

\section{The answering process}

1 Go to www.rcplondon.ac.uk/SAQ

2 Log on using your usual RCP username and password

3 Select the relevant $\mathrm{CME}$ question paper

4 Answer all 10 questions by selecting the best answer from the options provided

5 Once you have answered all the questions, click on Submit

\section{Registering your external CPD credits}

Carrying out this activity allows you to claim two external CPD credits. These will be automatically transferred to your CPD diary, where you can review the activity and claim your points.

1 Regarding dengue infection, which of the following are true?

(a) Diabetes and hypertension are risk factors for worse outcomes in dengue infections.

(b) Severe manifestations of dengue usually present in the first 48 hours of the illness.

(c) Steroids have been shown to be beneficial in patients with dengue shock.

(d) Thrombocytopaenia only occurs in severe dengue.

(e) Travel-associated dengue has not been reported from sub-Saharan Africa.

2 A 27-year-old man presented to the emergency department five days after returning from a holiday in the USA. He spent his time in Oklahoma on a one-week road trip, followed by a further one week of hiking and cycling around forested lakes. He travelled with three friends who all remained well. On presentation he was febrile and confused with no signs of meningism. On examination he had no lymphadenopathy but had a diffuse maculopapular rash over his torso. Neurological examination was normal apart from a mild tremor of his hands. His mother stated that he was previously fit and well, and had underwent no other foreign travel in the last 12 months. Of note, he had one dose of MMR vaccination in childhood, but never received the second dose. Which is the most likely diagnosis?

(a) Enterovirus 71 encephalitis

(b) HSV encephalitis

(c) Japanese encephalitis

(d) mumps encephalitis

(e) West Nile encephalitis

3 A 42-year-old woman, originally from Thailand but resident in the UK for the past two years presented to the emergency department with a two-day history of increasing lethargy, confusion and fever. On examination she had cervical, axillary and inguinal lymphadenopathy, and oral candidiasis, but no rash. Neurological examination revealed a VI cranial nerve palsy. Her husband stated she had not been back to Thailand since moving to the UK, and had no ill contacts. A CT brain performed in the emergency department was normal and a lumbar puncture was planned. In addition to biochemical analysis, microbiological culture and viral PCR of the CSF, which additional test is most likely to be helpful in her management?
(a) C-ANCA
(b) HIV serology
(c) lymph node biopsy
(d) malaria film
(e) MRI brain

4 A 52-year-old male with a past history of liver transplantation presented with a three-day history of fevers, mylagias, arthralgias and shortness of breath. He lived in a wooded area in Cape Cod, and remained on standard immunosuppression. Examination revealed pale conjunctivae and bibasal crepitations. Laboratory tests showed haemoglobin $10 \mathrm{~g} / \mathrm{dl}$ (12-14), serum creatinine $150 \mu \mathrm{mol} / \mathrm{l}(65-86)$, alanine transaminase (ALT) $82 \mathrm{IU} / \mathrm{I}$ $(<40)$ and aspartate transaminase (AST) 85 IU/I (<40). What is the most appropriate initial test?

(a) anaplasmosis/ehrlichiosis serology

(b) Babesia PCR 
(c) Giemsa-stained blood film to look for parasites

(d) Lyme serology

(e) Rocky Mountain Spotted Fever serology

5 A business man, who goes on short trips to India, asked if he should have the rabies vaccine. He was due to fly out in one week's time. What is the most appropriate recommendation?

(a) Short trips to Indian cities carry little risk, so vaccine is not necessary.

(b) One week is not enough time for a vaccine course, so come back at least a month before the next trip.

(c) He should have a dose of vaccine now and again just before he leaves. A third dose can be given in a few weeks' time.

(d) He should have a three-dose course of vaccine on days 0 , 3 and 7.

(e) Post-exposure treatment with vaccine and immune globulin will always be available if, when and where it is needed, so pre-exposure vaccine is not required.

6 A 33-year-old male backpacker arrived four days after returning to the UK from a 14-day safari in Tanzania. He presented with a four-day history of relapsing fever, headache, malaise and nausea. On examination there was a visible lesion (see picture) on his neck. Rapid diagnostic test for malaria was negative. Which investigation would be the most appropriate diagnostic test?

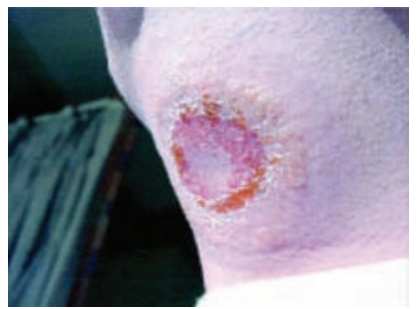

Reproduced with permission of Hospital Tropical Diseases. Previously published in Emerg Infect Dis 2002;8:74-6.

(a) lumbar puncture

(b) rickettsial serology

(c) skin biopsy

(d) serology for Rhodesiense HAT

(e) thick and thin blood films to look for malaria and trypanosomes

7 A 25-year-old British backpacker presented with a three-day history of fatigue with fever, diarrhoea and an urticiarial rash. On examination he had hepatosplenomegaly and a mild wheeze. He recently returned from a three-month trip to Tanzania and Malawi where he went on safari, swam in freshwater lakes, recalls numerous insect bites and ate/ drank local food and drink. On admission, his routine blood tests showed a normal haemoglobin and platelet count, but raised white cell count at $15.6 \times 10^{9} / /(3.5-9.0)$ (neutrophils $5.3 \times 10^{9} / /(1.3-5.4)$, lymphocytes $2.4 \times 10^{9} / /(0.7-3.5)$ and eosinophils $\left.7.9 \times 10^{9} / /(<0.4)\right)$. Three thick and thin blood films for malaria were negative.

What is the most likely diagnosis?
(a) dengue
(b) leishmaniasis
(c) malaria
(d) schistosomiasis
(e) trypanosomiasis

8 A 45-year-old British man presented to an emergency department in London with a two-day history of headache and nausea with fever and rigors. He had returned after three months of working in Sierra Leone eight days previously. He was a senior engineer for a mining company in the north-west of the country and had been going out to Sierra Leone intermittently for several years. He was based in a highly controlled compound for expatriate workers. He denied contact with anybody who was ill or with any dead bodies, and never saw any rats. He had had all appropriate immunisations before going to Sierra Leone but did not take any malaria prophylaxis. On examination he was alert, temperature was $38^{\circ} \mathrm{C}$, heart rate $110 \mathrm{bpm}$, blood pressure $100 / 78 \mathrm{mmHg}$, tongue was dry and he appeared mildly jaundiced. His abdomen was soft with no organomegaly. There was no evidence of rash, bleeding or bruising.

What is the single most important investigation to be performed next?
(a) clotting profile
(b) full blood picture
(c) renal and liver biochemistry
(d) malaria test
(e) test for Ebola RNA

9 Following exposure to Coxiella, which of the following most commonly occurs in the immunocompetent?
(a) asymptomatic infection
(b) headache
(c) hepatitis
(d) miscarriage
(e) respiratory upset

10 Infection with which of the following typically causes blood-stained diarrhoea?
(a) Clostridium difficile
(b) Cryptosporidium parvum
(c) Entamoeba histolytica
(d) Isospora belli
(e) Norovirus

\section{CME Oncology in the emergency} room SAQs

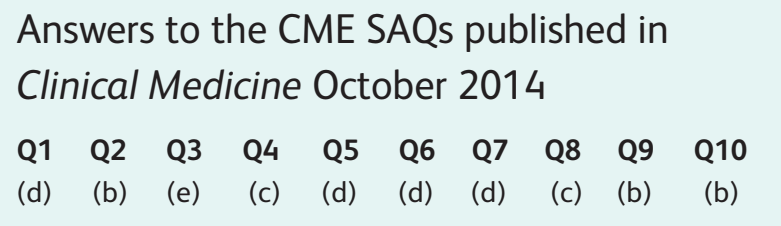

\section{CME Diabetes SAQs}

Answers to the CME SAQs published in Clinical Medicine December 2014
Q1
Q2 Q3
Q4
Q5
Q6 Q7
Q8 Q9
Q10
(c)
(e) (e)
(d) (b)
(d) (d)
(d) (a) 